\title{
AVALIAÇÃO POR COMPETÊNCIAS - UMA PROPOSTA DE APLICAÇÃO EM DISCIPLINAS DE ENGENHARIA
}

Rodrigo Cutri-rodrigo.cutri@maua.br

Instituto Mauá de Tecnologia

Praça Mauá, 1

09580-900 - São Caetano do Sul - SP

Hector Alexandre Chaves Gil - hector.gil@maua.br

Instituto Mauá de Tecnologia

Praça Mauá, 1

09580-900 - São Caetano do Sul - SP

Patrícia Antônio de Menezes Freitas - pantonio@maua.br

Instituto Mauá de Tecnologia

Praça Mauá, 1

09580-900 - São Caetano do Sul - SP

Resumo: A RESOLUÇÃO N ${ }^{\circ}$ 2, DE 24 DE ABRIL DE 2019 do Conselho Nacional de Educação que instituiu as Diretrizes Curriculares Nacionais do Curso de Graduação em Engenharia traz em grande destaque o desenvolvimento das competências do egresso ao longo de sua jornada acadêmica. Mas a grande questão permanece: como desenvolver um currículo por competências? Ou como aplicar o conceito de avaliação por competências de maneira prática dentro de uma estrutura curricular? Este artigo apresenta um procedimento para a implantação de avaliação baseada em competências a partir de referências bibliográficas relacionadas a cursos de Engenharia e a seguir apresenta o planejamento inicial de uma proposta de aplicação em algumas das disciplinas do Ciclo Básico de Engenharia do Instituto Mauá de Tecnologia.

Palavras-chave: DCN. Avaliação. Competências.

\section{INTRODUÇÃO}

Ao se observar atentamente o texto das novas Diretrizes Curriculares Nacionais para Engenharia (MEC, 2019), vemos que o termo "competência" aparece vinte e três vezes, tamanha a importância dada ao seu desenvolvimento e as competências que o egresso deve possuir para o mercado de trabalho. Mas o que é competência? Segundo o PAS (Publicly Available Specification) patrocinado pelo Conselho de Engenharia do Reino Unido, que originou o PAS 525:2018 -Framework for assessing professional engineering competence Specification [Engineering Council UK, 2018], os seguintes termos se relacionam à palavra competência:

- competência (adjetivo): capacidade de aplicar conhecimentos e habilidades de engenharia a alcançar os resultados pretendidos (ABNT, 2013)

- competência (substantivo): elemento que é medido para demonstrar competência 
- quadro de competências: conjunto de competências aplicáveis à engenharia.

- avaliação de competência: métodos sistemáticos de coleta de dados sob condições padronizadas para chegar a uma conclusão sobre a competência e o compromisso de um engenheiro

Esta são especificações harmonizadas internacionalmente para avaliar a competência e o comprometimento dos profissionais de engenharia, com base no padrão do Engineering Council UK para competência profissional em engenharia: UK-SPEC.

Mas, se competência é capacidade de aplicar conhecimentos e habilidades de engenharia a alcançar os resultados pretendidos, como podemos construir um currículo com o foco em competências ou promover a avaliação por competências em disciplinas visando as habilidades necessárias para o futuro profissional (OECD, 2019)?

Este artigo apresenta um procedimento para a implantação de avaliação por competências a partir de referências bibliográficas relacionadas à cursos de Engenharia e a seguir apresenta uma proposta de aplicação, em desenvolvimento, em algumas das disciplinas do Ciclo Básico de Engenharia do Instituto Mauá de Tecnologia (Físicas 1 e 2, Química e Fundamentos em Engenharia).

\section{EDUCAÇÃO BASEADA EM COMPETÊNCIAS}

A Educação por Competências é um modelo de educação que usa a aprendizagem, não o tempo, como a métrica do sucesso do aluno. Concentra-se em competências derivadas das habilidades comprovadamente mais relevantes por educadores e empregadores. Diversos estudos publicados em outros países tratam do desenho de currículo por competências (Mariana Leandro Cruz, et al, 2019), (Leyva, M. R. V., 2008), (Rojas Olivares, G. D. J. ,2015) e (Ruay R, Garcés J., 2015), das competências necessárias do corpo docente (Perrenoud, P., \& Thurler, M. G., 2009) e (Rajapaksha, A., \& Hirsch, A. S., 2017) e das formas de avaliação correlatas à avaliação por competências (Purzer, S., et al 2017), (George Mason University, 2019), (AAES, 2019), (C.Whitcomb, R. Khan, C. White, 2014), (Purdue University, 2019), (Balascio, C., \& Brumm, T., \& Mickelson, S., 2010) e (Dym, C. L, et al, 2006).

\subsection{Como aplicar uma Educação Baseada em Competências?}

Primeiramente deve-se estabelecer um objetivo educacional (Missão - aonde queremos chegar) e estabelecer metas (Linha do Tempo) para atingir este propósito. Assim, se o objetivo é que o estudante adquira uma determinada competência, a questão é definir quando ela deve ser atingida. Ao mesmo tempo, é fundamental definir quais os objetivos educacionais (usando a Taxonomia de Bloom) que se pretende alcançar e quais os resultados esperados dos estudantes. Para isso, deve-se ter indicadores de desempenho que permitam classificar os níveis de desempenho naquela determinada competência. A construção desses indicadores deve estar baseada em evidências. A estrutura curricular pode prever o desenvolvimento de determinada competência em diversas disciplinas com níveis crescentes de desenvolvimento, ou definir espaços de tempo próprio e contínuos para que aquela competência seja desenvolvida. 
Ao se falar em avaliação por competências, geralmente prevê-se uma avaliação por projetos e conduta. Ao se observar as novas DCNs de Engenharia pode-se de modo geral depreender as seguintes competências:

- criar e sintetizar conhecimento;

- dominar habilidades de comunicação escrita e oral ;

- pensar de forma crítica e reflexiva;

- demonstrar habilidades de engenharia;

- aplicar conhecimentos na resolução de problemas de maneira apropriada;

- desenvolver habilidades interpessoais (soft skills)

Quais seriam evidências de que o corpo discente obteve estas competências? Apresenta-se exemplos por meio do Quadro 1:

Quadro 1 - Propostas de Evidência de que o corpo discente obteve determinada competência

\begin{tabular}{|c|c|}
\hline Competência & Exemplo de Evidência \\
\hline $\begin{array}{l}\text { Desenvolver } \\
\text { conhecimento }\end{array}$ & $\begin{array}{l}\text { Realizar um projeto de pesquisa individual ou em colaboração com } \\
\text { colegas ou professores }\end{array}$ \\
\hline $\begin{array}{l}\text { Sintetizar } \\
\text { Conhecimento }\end{array}$ & $\begin{array}{l}\text { Escrever um artigo científico com base nas diretrizes de publicação } \\
\text { de um periódico nacional ou comparável } \\
\text { Escrever um relatório do projeto com base em um projeto de } \\
\text { pesquisa }\end{array}$ \\
\hline $\begin{array}{l}\text { Comunicar } \\
\text { Conhecimento }\end{array}$ & $\begin{array}{l}\text { Apresentar os resultados de um projeto de estudo na forma de um } \\
\text { seminário } \\
\text { Enviar um artigo para uma revista apropriada } \\
\text { Explicar soluções de problemas } \\
\text { Desenvolver materiais instrucionais que comuniquem informações } \\
\text { a diversos usuários finais }\end{array}$ \\
\hline $\begin{array}{l}\text { Pensar de forma } \\
\text { crítica e reflexiva }\end{array}$ & $\begin{array}{l}\text { Preparar um artigo crítico e reflexivo sobre tópicos específicos } \\
\text { Projeto de pesquisa com reflexão crítica sobre os métodos aplicados } \\
\text { e resultados obtidos }\end{array}$ \\
\hline $\begin{array}{l}\text { Demonstrar } \\
\text { habilidades } \\
\text { engenharia }\end{array}$ & $\begin{array}{l}\text { Passar em um exame de qualificação na disciplina } \\
\text { Concluir com êxito os mini-cursos apropriados sobre fundamentos } \\
\text { de Física com problemas de engenharia }\end{array}$ \\
\hline
\end{tabular}

Para que o objetivo educacional possa a partir da evidência ser então categorizado são necessários os indicadores de desempenho ou critérios de performance, as chamadas rubricas. As rubricas são uma ferramenta de pontuação ou classificação usada para medir o desempenho e o aprendizado dos estudantes em um conjunto de critérios e objetivos. Não há um conjunto unificado de rubricas porque as rubricas de pontuação variam de acordo com as diferentes disciplinas e cursos. Existem três componentes nas rubricas, a saber: 
(i) dimensões / critérios de performance: os aspectos de desempenho que serão avaliados,

(ii) descritores: características que estão associadas a cada dimensão e

(iii) escala / nível de desempenho: uma escala de classificação que define o nível de domínio dos alunos dentro de cada critério.

A figura 1, traduzida de (Chan, C., 2015). mostra as escalas e dimensões necessárias em uma rubrica.

Desenvolver rubricas é uma tarefa árdua e trabalhosa, sugere-se a inicialmente a adoção de sites como (Tech4Learning, 2019) que permitam o desenvolvimento de rubricas personalizadas a partir de um texto padrão, que depois pode ser adaptado à disciplina em que se pretende desenvolver a avaliação por competência.

Figura 1 - Escalas e dimensões necessárias em uma rubrica.

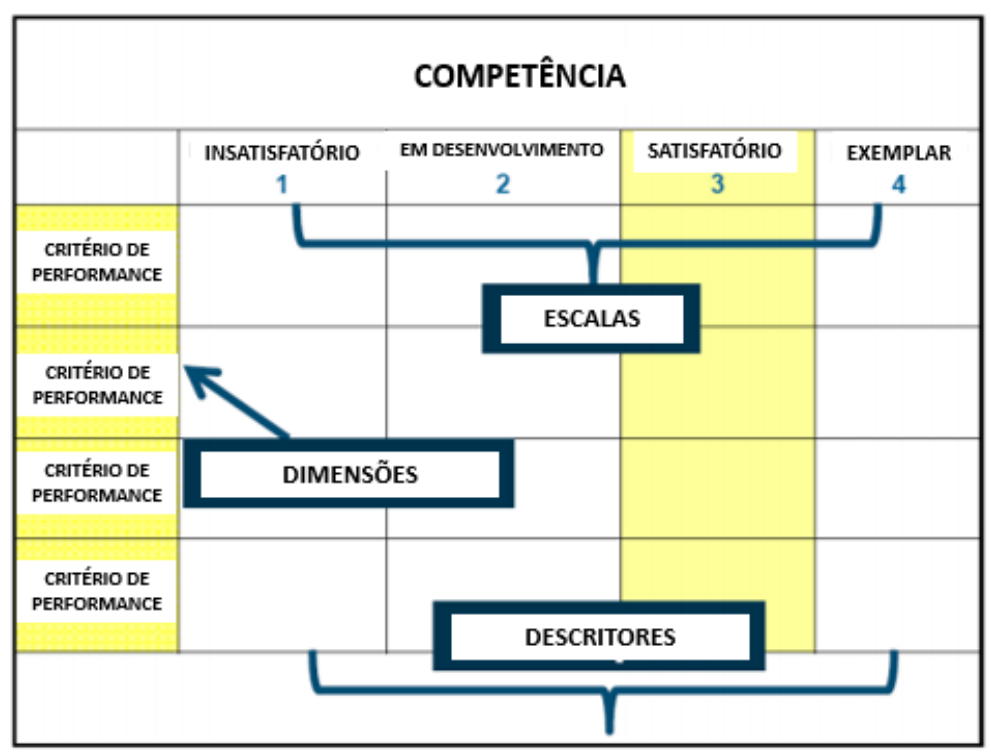

Desenvolver uma "rubrica", segue o seguinte procedimento:

$1^{\circ}$ Definição da competência à ser analisada

$2^{\circ}$ Definição dos critérios de performance que se deseja avaliar (objetivos educacionais)

$3^{\circ}$ Definição da evidência que será analisada

$4^{\circ}$ À partir do cruzamento da evidência com o critério de performance deve-se classificar o desempenho em níveis e atribuir descritores que permitam de modo mais objetivo diferenciar os níveis de proficiência que se deseja atribuir.

A rubrica deve ser explicitada ao estudante de modo que ele saiba como será avaliado e assim busque esforços para atingir seu máximo desempenho. 


\section{PROPOSTA DE AVALIAÇÃO POR COMPETENCIAS EM DESENVOLVIMENTO EM ALGUMAS DAS DISCIPLINAS INICIAIS DO CICLO BÁSICO DE ENGENHARIA NO INSTITUTO MAUÁ DE TECNOLOGIA.}

Desde 2017 (GIL, H. A. C., et al., 2017), o ciclo básico dos cursos de Engenharia do Centro Universitário do Instituto Mauá de Tecnologia conta com dois eixos estruturantes:

I - Motivacional e Formativos de Habilidades Transversais;

II - Profissional e Formativo de Habilidades Específicas,

Assim, a disciplina Fundamentos de Engenharia, conduz um eixo de trabalho profissional e formativo de habilidades específicas, com base em projetos conforme a Figura 2.

Figura 2 - Posicionamento da disciplina Fundamentos de Engenharia na $1^{\mathrm{a}}$ série e a organização das disciplinas e atividades no seu entorno.

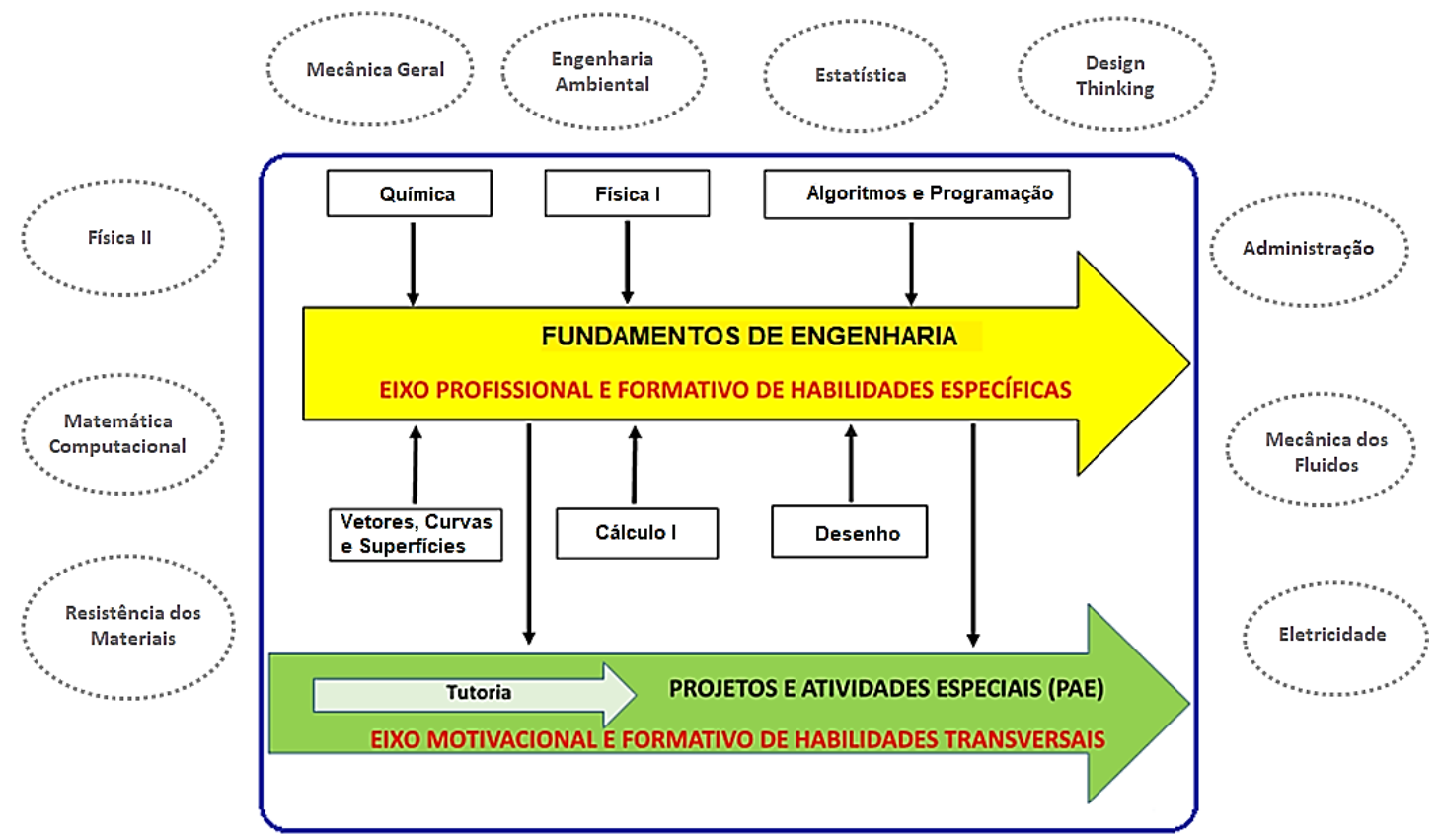

A disciplina Fundamentos de Engenharia assume assim um papel de linha mestra do $1^{\circ}$ ano do curso, tendo a importante função estruturante em que as disciplinas regulares atuam em conjunto.

As disciplinas do Ciclo Básico já vêm desenvolvendo a aplicação de diversas metodologias ativas de aprendizagem em suas aulas de teoria e laboratório como por exemplo: aprendizagem por problemas e projetos, peer-instruction dentre outras (Cutri, R. et al, 2016). Para 2020, pretende-se que algumas das atividades desenvolvidas pelos estudantes passem a usar de forma mais explicita a abordagem de avaliação por competências. Deste modo, frente as competências gerais esperadas na formação do Engenheiro (item 2.1) optou-se por buscar como evidência das competências esperadas, as disciplinas que já desenvolvem projetos semestrais elaborados por equipes de estudantes, geralmente compostas por 3 a 4 alunos (Cutri, R. et al, 2016) e (Lopes, R., et al 2018). Deste modo foram escolhidas como piloto as disciplinas: Física I e II, Química Geral e Fundamentos de Engenharia. 


\subsection{A análise e devolutiva por rubricas}

As disciplinas de Física I e II do currículo do Instituto Mauá de Tecnologia, fazem parte do ciclo básico de Engenharia e são aplicadas na forma de 2 aulas de 50 minutos de teoria e 2 aulas de 50 minutos de laboratório, com os seguintes objetivos educacionais, onde os estudantes devem demonstrar:

- que compreendem as formas de conhecimento científico, incluindo indutivo e dedutivo, empírico e teórico.

- a capacidade de desenvolver e testar uma hipótese.

- a capacidade de ler e interpretar dados.

- seu conhecimento dos métodos quantitativo e qualitativo.

- conhecimento do método científico.

- uma consciência da comunicação como parte integrante da apresentação de resultados científicos para a sociedade.

- a capacidade de entender e valorizar o papel da ciência na tomada de decisões.

Para a avaliação destas competências, por meio das evidências do projeto experimental desenvolvido em laboratório, foi elaborada uma rubrica por meio do site Rubric-Maker (Tech4Learning, 2019) - Quadro 2:

Quadro 2 - Exemplo de Rubrica à ser aplicada ao Projeto de Física

\begin{tabular}{|c|c|c|c|c|}
\hline & 4. Destaque & 3. Proficiente & 2. Aprendiz & 1. Iniciante \\
\hline $\begin{array}{l}\text { Avaliação do } \\
\text { trabalho de } \\
\text { laboratório: } \\
\text { identificar } \\
\text { fontes de erro } \\
\text { e efeito nos } \\
\text { resultados }\end{array}$ & $\begin{array}{l}\text { Identificou } \\
\text { possíveis fontes de } \\
\text { erro. Explicou como } \\
\text { os erros podem ter } \\
\text { distorcido os dados. } \\
\text { Considerou } \\
\text { significância dos } \\
\text { resultados e como o } \\
\text { experimento pode } \\
\text { ser modificado para } \\
\text { aumentar } \\
\text { validade. } \\
\text { Resultados } \\
\text { comparados } \\
\text { resultados } \\
\text { experiências } \\
\text { semelhantes. }\end{array}$ & $\begin{array}{l}\text { Identificou } \\
\text { possíveis fontes } \\
\text { de erro. Discutiu } \\
\text { como } \\
\text { experimento } \\
\text { pode } \\
\text { modificado para } \\
\text { aumentar } \\
\text { validade. }\end{array}$ & $\begin{array}{l}\text { Fontes de erro } \\
\text { identificadas } \\
\text { incorretamente. } \\
\text { As } \\
\text { modificações } \\
\text { propostas não } \\
\text { necessariamente } \\
\text { melhorariam os } \\
\text { resultados ou a } \\
\text { validade. }\end{array}$ & $\begin{array}{l}\text { Não identificou } \\
\text { fontes de erro. } \\
\text { Comentários e } \\
\text { modificações } \\
\text { propostas não } \\
\text { relevantes para } \\
\text { o experimento. }\end{array}$ \\
\hline $\begin{array}{l}\text { Métodos de } \\
\text { trabalho de } \\
\text { laboratório: } \\
\text { Descrever o }\end{array}$ & $\begin{array}{l}\text { A montagem foi } \\
\text { completamente } \\
\text { documentada. } \mathrm{O} \\
\text { método também foi }\end{array}$ & $\begin{array}{lr}\text { A montagem } \\
\text { teve um texto } \\
\text { descritivo } \\
\text { diagramas. }\end{array}$ & $\begin{array}{l}\text { A descrição foi } \\
\text { geral ou não } \\
\text { incluiu } \\
\text { diagramas. }\end{array}$ & $\begin{array}{l}\text { A montagem } \\
\text { não foi descrita } \\
\text { ou } \\
\text { documentada. }\end{array}$ \\
\hline
\end{tabular}


(C) COBENCE 2020

"Os desafios para formar hoje o engenheiro do amanhã"

\begin{tabular}{|c|c|c|c|c|}
\hline $\begin{array}{l}\text { processo e a } \\
\text { montagem. }\end{array}$ & $\begin{array}{l}\text { documentado de } \\
\text { forma completa e } \\
\text { precisa, facilitando } \\
\text { a reprodução do } \\
\text { experimento. }\end{array}$ & $\begin{array}{l}\text { experiência } \\
\text { pode ser } \\
\text { reproduzida } \\
\text { usando as etapas } \\
\text { fornecidas. }\end{array}$ & $\begin{array}{l}\text { Estavam } \\
\text { faltando várias } \\
\text { etapas no } \\
\text { procedimento. } \\
\text { As informações } \\
\text { fornecidas não } \\
\text { são suficientes } \\
\text { para replicar o } \\
\text { experimento. }\end{array}$ & $\begin{array}{l}\text { O passo a passo } \\
\text { do } \\
\text { procedimento } \\
\text { estava ausente } \\
\text { ou inadequado. }\end{array}$ \\
\hline $\begin{array}{l}\text { Visão geral } \\
\text { do trabalho } \\
\text { de } \\
\text { laboratório: } \\
\text { Método } \\
\text { científico: } \\
\text { observação, } \\
\text { hipótese, } \\
\text { previsão, } \\
\text { experimento, } \\
\text { conclusão }\end{array}$ & $\begin{array}{lr}\text { A hipótese } & \text { foi } \\
\text { baseada } & \text { na } \\
\text { observação. } & \text { Foi } \\
\text { realizada } & \text { uma } \\
\text { previsão e } & \text { foi } \\
\text { testada. } & \text { Foram } \\
\text { escritas conclusões } \\
\text { sobre hipóteses com } \\
\text { base no } \\
\text { experimento. Se a } \\
\text { hipótese era falsa, } \\
\text { foi alterada e um } \\
\text { novo experimento } \\
\text { foi proposto. }\end{array}$ & $\begin{array}{l}\text { Foram } \\
\text { realizadas } \\
\text { observações } \\
\text { claras e se } \\
\text { escreveu uma } \\
\text { hipótese. } \\
\text { realizada uma } \\
\text { previsão e foi } \\
\text { testada. Foram } \\
\text { escritas } \\
\text { conclusões } \\
\text { sobre hipóteses } \\
\text { com base no } \\
\text { experimento. }\end{array}$ & $\begin{array}{l}\text { A hipótese } \\
\text { respondeu ao } \\
\text { problema } \\
\text { observado. Foi } \\
\text { realizada uma } \\
\text { previsão. Não se } \\
\text { conseguiu } \\
\text { relacionar a } \\
\text { hipótese com o } \\
\text { experimento. }\end{array}$ & $\begin{array}{l}\text { A hipótese não } \\
\text { respondeu ao } \\
\text { problema } \\
\text { observado. Não } \\
\text { fez uma } \\
\text { previsão. O } \\
\text { experimento } \\
\text { não respondeu } \\
\text { à previsão ou } \\
\text { se relacionou } \\
\text { com a hipótese. }\end{array}$ \\
\hline $\begin{array}{l}\text { Visão geral } \\
\text { do trabalho } \\
\text { em equipe: } \\
\text { ideias } \\
\text { ouvidas e } \\
\text { compartilhad } \\
\text { as, membros } \\
\text { da equipe } \\
\text { apoiados, } \\
\text { problemas } \\
\text { discutidos, } \\
\text { trabalho } \\
\text { próprio } \\
\text { concluído }\end{array}$ & $\begin{array}{l}\text { Ouviu atentamente } \\
\text { o que os outros } \\
\text { disseram. } \\
\text { Compartilhou ideias } \\
\text { e recursos } \\
\text { livremente. } \\
\text { Conversou sobre } \\
\text { problemas } \\
\text { encontrou soluções } \\
\text { úteis. Concluiu o } \\
\text { próprio trabalho no } \\
\text { prazo e forneceu } \\
\text { ajuda a outros } \\
\text { membros da equipe. }\end{array}$ & 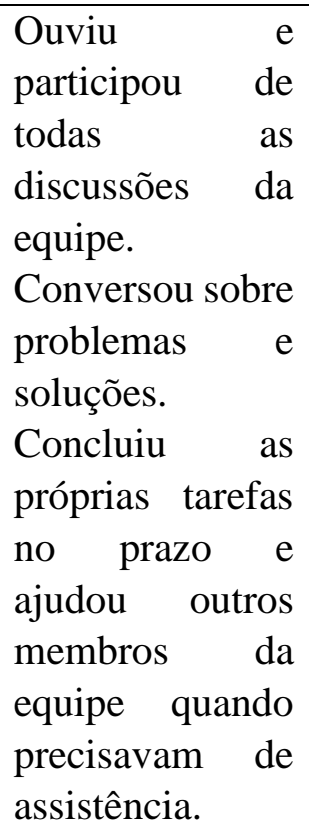 & $\begin{array}{lr}\text { Ouviu } & \mathrm{e} \\
\text { participou } & \text { de } \\
\text { algumas } & \\
\text { discussões } & \text { em } \\
\text { equiper } & \text { Falou } \\
\text { mais } & \text { sobre } \\
\text { problemas do } & \text { que soluções. } \\
\text { Concluiu as } \\
\text { tarefas } \\
\text { atribuídas, mas } \\
\text { não ajudou } \\
\text { outros membros } \\
\text { da equipe. }\end{array}$ & $\begin{array}{l}\text { Raramente } \\
\text { ouvia ou } \\
\text { participava de } \\
\text { discussões em } \\
\text { equipe. Falou } \\
\text { apenas sobre } \\
\text { problemas, não } \\
\text { soluções. Não } \\
\text { concluiu todas } \\
\text { as tarefas } \\
\text { atribuídas. Não } \\
\text { ajudou outros } \\
\text { membros da } \\
\text { equipe. }\end{array}$ \\
\hline
\end{tabular}


Esta rubrica será aplicada as equipes de projeto de Física I e II no $1^{\circ}$ e $2^{\circ}$ semestres de 2020 , permitindo-se que o aluno tenha a devida devolutiva e possa assim reconhecer seus pontos de melhoria e promover ações para aperfeiçoá-los. Rubrica semelhante com as devidas adaptações às disciplinas serão aplicadas no projeto desenvolvido por Fundamentos de Engenharia e Química Geral.

\section{CONSIDERAÇÕES FINAIS}

A avaliação por competências é uma prática inovadora que deve ser assimilada pelo corpo discente e docente para que possa ser trabalhada de forma adequada. O planejamento inicial das atividades à serem desenvolvidas dá um arcabouço teórico-prático de como aplicar este conceito nos cursos de Engenharia.

Ao desenvolver as rubricas para cada disciplina vai-se formando um melhor entendimento do próprio corpo docente e discente de quais são as competências que se pretende atingir e quais as evidências necessárias para que sejam efetivamente buscadas.

\section{REFERÊNCIAS}

AAES - AMERICAN ASSOCIATION OF ENGINEERING SOCIETIES - ENGINEERING COMPETENCY MODEL - Disponível em: http://www.aaes.org/model - Acesso em: 29 nov. 2019.

ASSOCIAÇÃO BRASILEIRA DE NORMAS TÉCNICAS. NBR ISO/IEC 17024:2013 Avaliação da conformidade - Requisitos gerais para organismos que certificam pessoas Disponível em: https://www.abntcatalogo.com.br/norma.aspx?ID=306579 - Acesso em: 29 nov. 2019.

BALASCIO, C.; BRUMM, T.; MICKELSON, S. (2010, June), Competency Based Assessment Of Engineering Technology Program Outcomes Paper presented at 2010 Annual Conference \& Exposition, Louisville, Kentucky. https://peer.asee.org/16100

WHITCOMB, C.; KHAN, R.; WHITE, C. Development of a System Engineering Competency Career Development Model: an analytical approach using Bloom's Taxonomy - 2014 - Disponível em: calhoun.nps.edu - Acesso em: 29 nov. 2019.

CHAN, Cky. "Rubrics for Engineering Education", Engineering Education Enhancement and Research Asia (E3R Asia), 2015. - Disponível em: https://hke3r.cetl.hku.hk/pdf/Rubricsfor-Engineering-Education.pdf - - Acesso em: 29 nov. 2019.

CRUZ, M.L.; SAUNDERS-SMITS, G.N.; GROEN, P. (2019): Evaluation of competency methods in engineering education: a systematic review, European - Journal of Engineering Education, DOI: 10.1080/03043797.2019.1671810

CUTRI, R.; et al. Ten Ways to Improve Learning Physics as Part of an Engineering Course. ASEE Annual Conference \& Exposition, 2016, New Orleans. Anais. Louisiana, 2016. 
DYM, C. L.; et al. Engineering design thinking, teaching, and learning. IEEE Engineering Management Review, 34(1), 65-92, 2006.

Engineering Council UK - PAS 525:2018 - Framework for assessing professional engineering competence - $\quad$ Specification - Disponível em: https://shop.bsigroup.com/ProductDetail/?pid=000000000030366570 Acesso em: 29 nov. 2019.

GEORGE MASON UNIVERSITY Assessment of Student Learning Template - Disponível em: https://www.schev.edu/docs/default-source/about-section/committees-andgroups/virginia-assessment-group/4year/assessgmu.pdf - Acesso em: 29 nov. 2019.

GIL, H. A. C.; Introdução à Engenharia como disciplina estruturante do primeiro ano de um curso de engenharia. Anais XLV COBENGE - Congresso Brasileiro de Educação em Engenharia: UDESC - JOINVILLE/SC, 2017

LEYVA, M. R. V. Diseño curricular por competencias. Asociación Nacional de Facultades y Escuelas de Ingeniería, 2008.

LOPES, R. D.; et al. Iniciativas inovadoras em disciplinas de introdução à engenharia. In: Adriana Maria Tonini;Tânia Regina Dias Silva Pereira. (Org.). DESAFIOS DA EDUCAÇÃo EM ENGENHARIA: Inovação e Sustentabilidade, Aprendizagem Ativa e Mulheres na Engenharia. 1ed.: ABENGE - Associação Brasileira de Educação em Engenharia, 2018, v. 1, p. 12-68.

MINISTÉRIO DA EDUCAÇÃO - RESOLUÇÃO No 2, DE 24 DE ABRIL DE 2019 - Institui as Diretrizes Curriculares Nacionais do Curso de Graduação em Engenharia. Disponível em: http://portal.mec.gov.br/component/content/article?id=12991 Acesso em: 29 nov. 2019.

OECD Future of Education and Skills 2030 - Conceptual learning framework - Disponível em: http://www.oecd.org/education/2030-project/about/documents/ - Acesso em: 29 nov. 2019.

PERRENOUD, P., \& THURLER, M. G. (2009). As competências para ensinar no século XXI: a formação dos professores e o desafio da avaliação. Artmed Editora.

PURDUE UNIVERSITY - Engineering Education Graduate Competencies - Disponível em: https://engineering.purdue.edu/ENE/Academics/Graduate/Competencies - Acesso em: 29 nov. 2019.

PURZER, Senay; et al., "An Assessment Framework for First-Year Introduction to Engineering Courses" School of Engineering Education Faculty Publications. Paper 16. 2017.

RAJAPAKSHA, A., \& HIRSCH, A. S. Competency based teaching of college physics: The philosophy and the practice. Physical Review Physics Education Research, 13(2), 020130. 2017. 
ROJAS OLIVARES, G. D. J. Medición de habilidades profesionales en estudiantes de Ingeniería Industrial de la Universidad de Chile. 2015. - Disponível em: http://repositorio.uchile.cl/handle/2250/137718 - Acesso em: 29 nov. 2019.

RUAY R, GARCÉS J. Diseño y construcción de instrumentos de evaluación de aprendizajes y Competências. RedIberoamericana de Pedagogía Colombia. 2015 Disponível em: https://institutoprofesionalmr.org/wp-content/uploads/2018/04/RuayGarc\%C3\%A9s-2015-Dise\%C3\%B1o-y-construcci\%C3\%B3n-de-instrumentos-deevaluaci\%C3\%B3n-de-aprendizajes-y-competencias.pdf - Acesso em: 29 nov. 2019.

TECH4LEARNING - Rubric maker Make customized assessments for student work. Disponível em: https://rubric-maker.com/ - Acesso em: 29 nov. 2019.

\title{
COMPETENCE ASSESSMENT - A PROPOSAL FOR APPLICATION IN ENGINEERING DISCIPLINES
}

\begin{abstract}
RESOLUTION No. 2, OF APRIL 24, 2019 of the National Council of Education that instituted the National Curriculum Guidelines for the Undergraduate Engineering Course brings out the development of the graduate's competences throughout his academic journey. But the big question remains: how to develop a competency-based curriculum? Or how to apply the concept of competency assessment in a practical way within a curriculum structure? This article presents a procedure for the implementation of competency-based assessment based on bibliographic references related to the Engineering Schools and then presents the initial planning of an application proposal in some disciplines of the Basic Engineering Cycle of the Mauá School of Engineering.
\end{abstract}

Key-words: DCN, Competence, Assessment. 